\title{
Primay Extraosseous Ewing's Sarcoma Of The Lombar Epidural Space Presenting As Cauda Equina Syndrome
}

Hatim Belfquih*, Brahim El mostarchid

Department of Neurosurgery, Mohammed V Military Teaching Hospital, Rabat, Morocco

Received: October 11, 2016; Accepted: October 28, 2016; Published: November 21, 2016

*Corresponding author: Hatim Belfquih, Department of Neurosurgery, Mohammed V Military Teaching Hospital, N²73, Hay Al Khiam III, Temara, Morocco, Tel: +212661338623; E-mail: hatimbelfquih@gmail.com

A 31-year-old woman complained of right sciatica for 2 months followed one month later by gait disturbance and weakness of right lower extremity. Magnetic Resonance Imaging (MRI) revealed a extradural mass extending from L4 to S1/S2 interspace. It appeared hypo-intense on T1-weighted and T2weighted images and contrast enhancement after injection of gadolinium (Figure 1A, B and C). Axial MR images revealed a mass extention to the right neural foramen at the L5-S1 which is enlarged (Fig1D, E). The patient underwent laminectomy of L4 to S1, A highly vascular lesion was seen in the epidural space, extending from $\mathrm{L} 4$ root axilla down to the $\mathrm{S} 1$ root, could be excised totally. there was no visible bone involvement. Histopathological study revealed high cellularity small round cells with hight nuclear cytoplasmic ratio (Figure 2). Immunohistochemically,

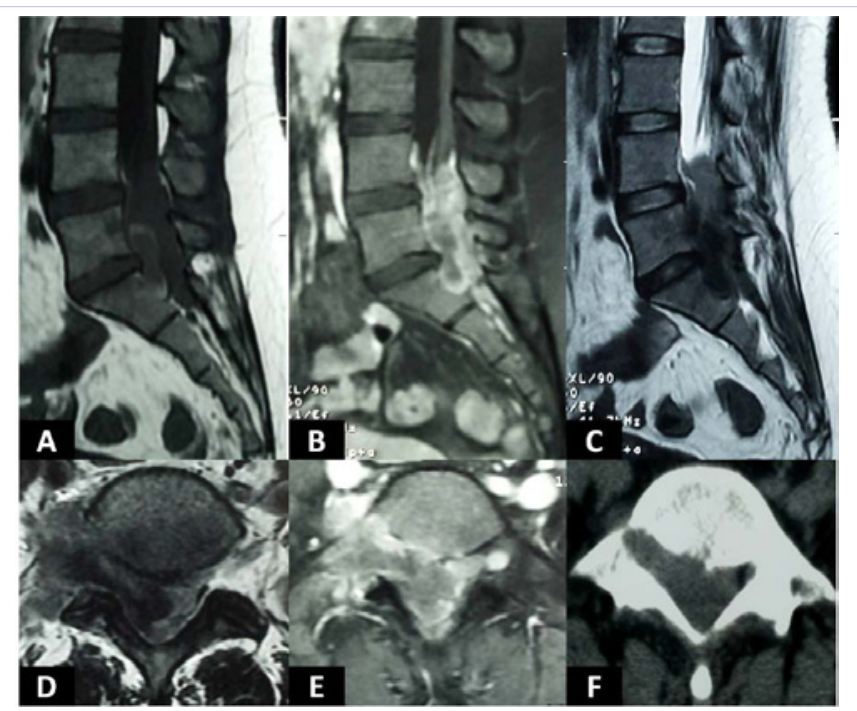

Figure 1: Precontrast (A) and postcontrast (B) sagittal T1-weighted and T2-weighted (C) MR images demonstrate a large extradural mass, measuring $8 \times 4 \times 2 \mathrm{~cm}$, extending from uper edge of L4 down to S1/S2 interspace. Hypo-intense on both T1-weighted and T2-weighted images and contrast enhancement after injection of gadolinium. Axial MR images : postcontrast T1-weighted (D) , T2-weighted (E) and axial CT scan (F) revealed the extension of the mass to the right neural foramen at the L5-S1 level which is enlarged.

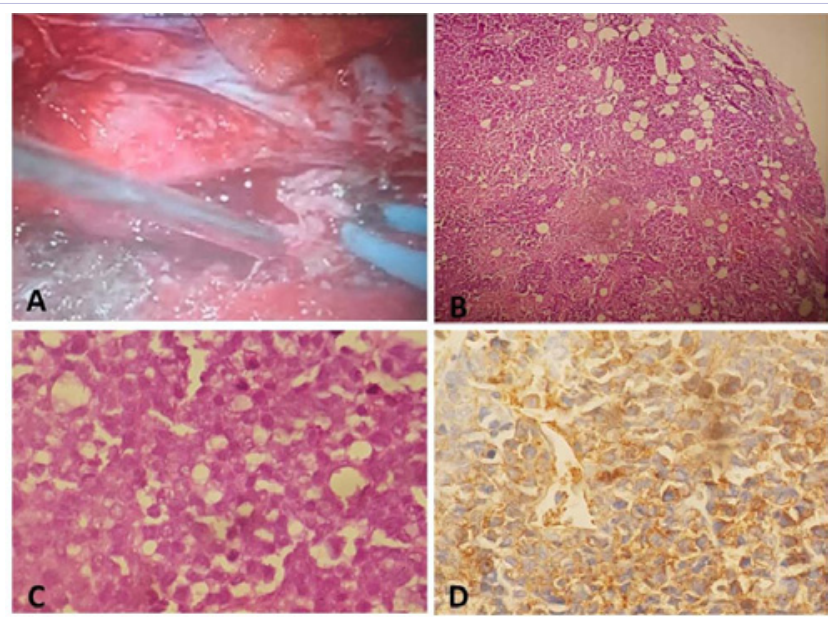

Figure 2: (A) Intraoperative photograph showing a hight vascular tumor along the lateral aspect of thecal sac and right S1 nerve root. (B and C) Hematoxylin and Eosin staining of the tumor under low- and high-power microscopic fields showing tumor cells with high $\mathrm{N}: \mathrm{C}$ ratio, stippled chromatin, vacuolated cytoplasm, and high mitotic activity. The tumor cells were diffusely immunoreactive to CD99 (X400) (D).

the tumor cells were positive for CD99. A diagnosis of Primary Extraosseous Ewing Sarcoma (EES) was favored and confirmed by molecular cytogenetic analysis. At 1-year follow-up, after completing adjuvant chemoradiotherapy, the patient is symptom free.

EES is a rare neoplasm that is difficult to diagnose. It can cause spinal cord and cauda equina compression [1]. Complete tumor resection with adequate chemoradiotherapy is considered as the optimal therapeutic [2].

\section{References}

1. Gopalakrishnan CV, Shrivastava A, Easwer HV, Nair S. Primary Ewing's sarcoma of the spine presenting as acute paraplegia. J Pediatr Neurosci. 2012;7(1):64-6. doi: 10.4103/1817-1745.97630.

2. Sade R, Cakir M, Ogul H, Yuce I, Kantarci M. Primary extraosseous Ewing sarcoma of the lombar spine presenting as left leg weakness. Spine J. 2015;15(6):1488-9. doi: 10.1016/j.spinee.2015.02.029. 